\title{
Baryon oscillations in galaxy and matter power-spectrum covariance matrices
}

\author{
Mark C. Neyrinck ${ }^{1}$ and István Szapudi ${ }^{1}$ \\ ${ }^{1}$ Institute for Astronomy, University of Hawaii, Honolulu, HI 96822, USA \\ email: neyrinckeifa.hawaii.edu. The definitive version is available at www.blackwell-synergy.com.
}

23 October 2018

\begin{abstract}
We investigate large-amplitude baryon acoustic oscillations (BAO's) in off-diagonal entries of cosmological power-spectrum covariance matrices. These covariance-matrix BAO's describe the increased attenuation of power-spectrum BAO's caused by upward fluctuations in largescale power. We derive an analytic approximation to covariance-matrix entries in the BAO regime, and check the analytical predictions using $N$-body simulations. These BAO's look much stronger than the BAO's in the power spectrum, but seem detectable only at about a onesigma level in gigaparsec-scale galaxy surveys. In estimating cosmological parameters using matter or galaxy power spectra, including the covariance-matrix BAO's can have a severalpercent effect on error-bar widths for some parameters directly related to the BAO's, such as the baryon fraction. Also, we find that including the numerous galaxies in small haloes in a survey can reduce error bars in these cosmological parameters more than the simple reduction in shot noise might suggest.
\end{abstract}

Key words: cosmology: theory - large-scale structure of Universe

\section{INTRODUCTION}

Humans are fortunate that there are baryons in the Universe. Not only are we made of them, but baryons are responsible for features in the shape of cosmological power spectra and two-point correlation functions that are quite valuable to cosmologists. These ' features are called baryon acoustic oscillations (BAO's), and are imprints of acoustic oscillations in the gas of the early universe (Peebles \& Yu 1970; Sunvaev \& Zel'dovich 1970; Holtzman 1989; Eisenstein \& Hu 1998; Meiksin. White \& Peacock 1999). Their presence in galaxy clustering statistics provides a standard ruler to measure the relation between distance and redshift, and the expansion of the universe at late times (Blake \& Glazebrook 2003; Seo \& Eisenstein 2003). They provide one of the main tools currently proposed for studying the effects of dark energy.

BAO's have been detected in modern low-redshift galaxy surveys, both in the 2dFGRS (Cole et al. 2005) and SDSS (Eisenstein et al. 2005; Hütsi 2006; Percival et al. 2007a). These detections were made using the (two-point) correlation function, or its Fourier dual, the power spectrum. Conveniently, the BAO regime is on large-enough scales that non-linear effects are mild. Still, for precision cosmology, these mild effects must be understood, and are the topic of much recent research (e.g. Springel et al. 2005; Jeong \& Komatsu 2006; Huff et al. 2006; Schulz \& White 2006; Angulo et al. 2007; Seo \& Eisenstein 2007; Smith, Scoccimarro \& Sheth 2007b).

Non-linear evolution of the matter power spectrum tends to dampen or smear BAO's. For example, Eisenstein, Seo \& White (2007) found that large-scale bulk flows and cluster formation produce motions that smear out the BAO peak in the correlation function, but that these motions are confined to relatively small scales of $\sim 10 h^{-1} \mathrm{Mpc}$ in Lagrangian space. They argued that these motions roughly preserve wiggles on the largest scales of the power spectrum, but wipe out wiggles on smaller scales. The effects we describe in this paper, using Eulerian perturbation theory, likely arise physically from the same large-scale bulk motions. The attenuation of BAO's can also be understood by considering an additive mode-coupling power spectrum, which rises on small scales as structure develops, along with a function which attenuates the linear power spectrum on non-linear scales. In the halo model (HM, reviewed in Cooray \& Sheth 2002), this small-scale contribution is the one-halo (1h) term, and comes from pairs of objects within single haloes. A qualitatively similar effect occurs in renormalized perturbation theory (RPT; Crocce \& Scoccimarro 2007; McDonald 2007), which is less empirical than the HM, and seems more accurate through translinear scales. For example, Crocce \& Scoccimarro (2007) show that, more physically than in the HM, the mode-coupling power spectrum in RPT goes to zero on small scales.

In this paper, we show that wiggles exist in off-diagonal terms of matter and galaxy power spectrum covariance matrices, almost entirely out-of-phase with BAO's in the power spectrum. We interpret these BAO's in the covariance matrix as manifestations of the suppression exacted on power-spectrum BAO's by power on large scales. Regions of the Universe with upward fluctuations in largescale power have more-suppressed BAO's.

The body of the paper is organized as follows. In Section 2 , we 


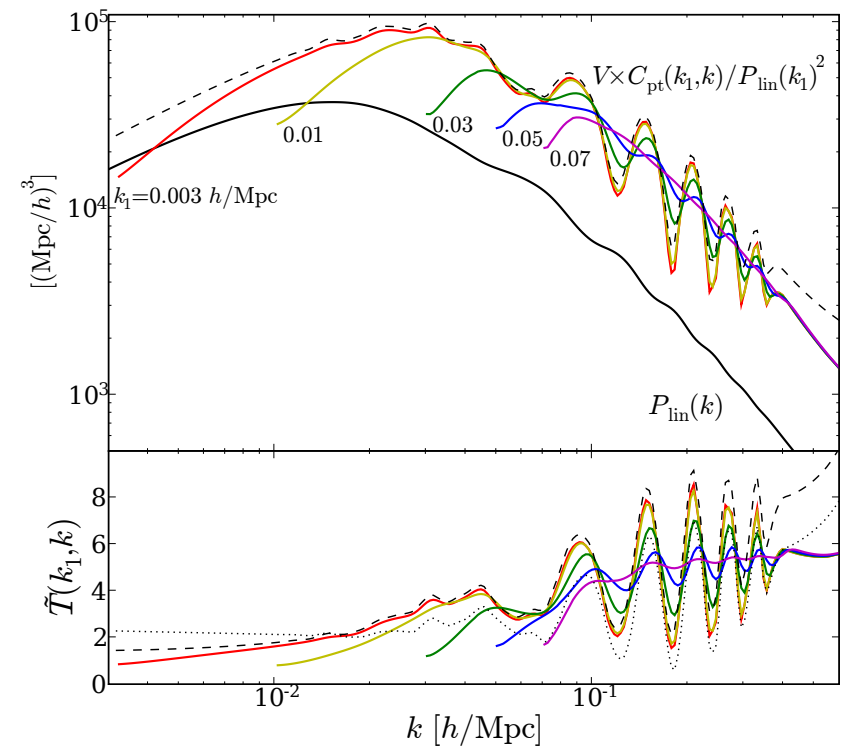

Figure 1. Baryon acoustic oscillations in off-diagonal terms of the powerspectrum covariance matrix, calculated using leading-order perturbation theory for the trispectrum. In the top panel, each colored curve is a row of the covariance matrix, divided by $P_{\text {lin }}\left(k_{1}\right)^{2}$, where $k_{1}$ is the wavenumber of that row. In the bottom panel, the curves are additionally divided by $P_{\text {lin }}(k)$. The dashed curves show the approximation of Eq. 3. The dotted curve in the bottom panel shows the approximation without the firstderivative term.

discuss analytic predictions for the BAO's in the covariance matrix of matter. In Section 3 , we test the analytic predictions against $N$ body simulations, and investigate the detectability of the wiggles in the covariance matrix. Finally, in Section 4 we investigate the effect of these covariance-matrix BAO's on cosmological parameter estimation, focusing on parameters directly related to BAO's in the power spectrum. We perform this analysis for both matter and galaxy power spectra, using the HM framework.

\section{ANALYTIC PREDICTIONS FOR COVARIANCE-MATRIX WIGGLES}

The covariance of the matter power spectrum in a survey of volume $V$ (neglecting survey-shape effects) is the sum of a Gaussian term, which depends on the square of the non-linear power spectrum, and a term involving the matter trispectrum (e.g. Scoccimarro, Zaldarriaga \& Hui 1999; Hamilton, Rimes \& Scoccimarro 2006, HRS).

$C_{i j}=\frac{1}{V}\left[\frac{(2 \pi)^{3}}{V_{s, i}} 2 P\left(k_{i}\right)^{2} \delta_{i j}+T_{i j}\right]$,

where $V_{s, i}$ is the volume of shell $i$ in Fourier space (proportional to $k_{i}^{3}$ for logarithmically spaced bins), and $T_{i j}$ is the parallelogramconfiguration trispectrum averaged over shells $i$ and $j$;

$$
\begin{aligned}
T_{i j} & \equiv C\left(k_{i}, k_{j}\right)_{i \neq j} \\
& \equiv \int_{s, i} \int_{s, j} T\left(\boldsymbol{k}_{i},-\boldsymbol{k}_{i}, \boldsymbol{k}_{j},-\boldsymbol{k}_{j}\right) \frac{d^{3} \boldsymbol{k}_{i}}{V_{s, i}} \frac{d^{3} \boldsymbol{k}_{j}}{V_{s, j}} .
\end{aligned}
$$

Figure 1 shows the dimensionless, normalized powerspectrum covariance $\tilde{T}\left(k_{1}, k\right) \equiv C\left(k_{1}, k\right) V /\left[P\left(k_{1}\right)^{2} P(k)\right]$, for various $k_{1}$ 's. The wiggles in $\tilde{T}$ are much stronger than in the power spectrum itself; the ratio of peak to trough can be nearly a factor of ten. However, as we discuss in Sect. 3.1 the amount of noise in a measurement of $\tilde{T}$ is also large.

For Fig. 1] we use $P_{\text {lin }}$ for the power spectrum $P$, and the leading-order (third-order) PT trispectrum for $T_{i j}$. In the upper panel, the curves are shown at redshift $z=0$; the curves in the lower panel are independent of power-spectrum normalization and redshift. This is because the PT trispectrum involves three powers of $P_{\text {lin }}(k)$, evaluated at different $k$ 's, as does the denominator in the definition of $\tilde{T}$. We use a linear power spectrum from CAMB ${ }^{1}$ (Lewis, Challinor \& Lasenby 2000), with the same $\Lambda$ CDM cosmology as assumed for the Millennium simulation (Springel et al. 2005): the Hubble constant $H_{0}=73 \frac{\mathrm{km}}{\mathrm{s} \mathrm{Mpc}}, \Omega_{\mathrm{CDM}}=0.205$, $\Omega_{b}=0.045$, the scalar spectral index $n_{s}=1$, and the rms density fluctuation in spheres of radius $8 \mathrm{~h} \mathrm{Mpc}^{-1}$ is $\sigma_{8}=0.9$. The dashed curve is an approximation for $k_{1} \ll k$, given by

$\tilde{T}\left(k_{1} \rightarrow 0, k\right) \approx \frac{5038}{2205}-\frac{36}{35} \frac{P_{\text {lin }}^{\prime}(k) k}{P_{\text {lin }}(k)}+\frac{1}{5} \frac{P_{\text {lin }}^{\prime \prime}(k) k^{2}}{P_{\text {lin }}(k)}$.

The dotted curve shows this approximation without the firstderivative term. See Appendix Afor a derivation of Eq. (3).

For $k_{1} \ll k$, the dominant terms for a $\Lambda$ CDM power spectrum are the second-derivative term, which produces wiggles $180^{\circ}$ outof-phase with the wiggles in $P_{\text {lin }}(k)$, and the constant. The firstderivative term shifts the wiggles slightly. The dominance of the second-derivative term over the first-derivative term implies that the main effect of large-scale power is that of suppressing powerspectrum BAO's, not moving them.

By analogy with the non-linear power spectrum relative to the linear power spectrum, it might seem that BAO's in the non-linear $\tilde{T}$ could be significantly attenuated on small scales compared to the PT prediction. However, leading order for the trispectrum is 3rd-order, not 1st-order as for the power spectrum; thus, the PT trispectrum should be valid into the mildly non-linear regime. At $z=0$, 3rd-order PT works to $1 \%$ for the power spectrum for $k \lesssim$ $0.065 \mathrm{~h} \mathrm{Mpc}^{-1}$, while the linear power spectrum fails at the $\sim 1 \%$ level already at $k \approx 0.01 \mathrm{~h} \mathrm{Mpc}^{-1}$ (Matsubara 2007).

On small scales, the PT trispectrum prediction is expected to fail. Probably the most plausible model currently on small scales is the halo model, the matter trispectrum of which was worked out by Cooray \& Hu (2001, CH).

Figure 2 compares the HM and PT predictions for $\tilde{T}$. We continue to use $P_{\text {lin }}$ in the denominator of the definition $\tilde{T}\left(k_{1}, k\right)=$ $C\left(k_{1}, k\right) V /\left[P\left(k_{1}\right)^{2} P(k)\right]$, for easy comparison of the pure PT prediction. Alternatively, $\tilde{T}$ could be defined with the halo-model matter power spectrum in the denominator. For the HM prediction, we use the same implementation as in Neyrinck \& Szapudi (2007, NS); some details of the implementation also appear in Appendix B below. At $z=0$, the three-halo (3h) term contributes significantly on large scales. This cannot be the case at arbitrarily small $k$, where PT should hold exactly. This extra contribution in the trispectrum over PT was noted by $\mathrm{CH}$; it is similar to the shot-noise-like one-halo term that contributes unphysical power on large scales of the power spectrum (Cooray \& Sheth 2002; Smith et al. 2003; Crocce \& Scoccimarro 2007). However, it could still be that the $3 \mathrm{~h}$ contribution is real at some mildly non-linear scale, such as in the BAO regime. 


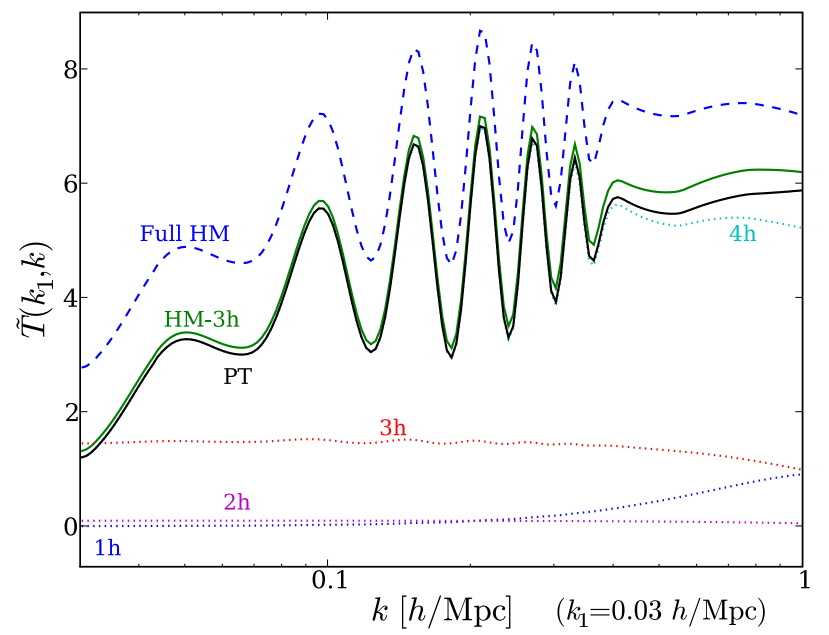

Figure 2. A comparison of different terms in the halo-model prediction for the normalized power-spectrum covariance $\tilde{T}\left(k_{1}, k\right)=$ $C\left(k_{1}, k\right) V /\left[P_{\text {lin }}\left(k_{1}\right)^{2} P_{\text {lin }}(k)\right]$ at $z=0$. We use $P_{\text {lin }}$ instead of the halo-model matter $P$ in the denominator to show clearly how $C\left(k_{1}, k\right)$ differs from the perturbation-theory prediction. On large scales, the $1 \mathrm{~h}$ and $2 \mathrm{~h}$ terms are negligible, but the $3 \mathrm{~h}$ term gives a probably inaccurate additional contribution. On smaller scales (both larger $k_{1}$ and $k$ ) than those shown, the $1 \mathrm{~h}$ term comes to dominate $C\left(k_{1}, k\right)$.

\section{TESTING AGAINST $N$-BODY SIMULATIONS}

To test the analytic predictions, we ran a large suite of $N$-body simulations to get a high signal-to-noise covariance matrix. In particular, we wanted to test our expectation that the added covariance from the HM $3 \mathrm{~h}$ term on large scales is artificial, and also to test the detectability of the wiggles, since their amplitude seems much larger than the wiggles in the power spectrum itself.

We ran 100 particle-mesh (PM) dark-matter simulations of box size $1024 h^{-1} \mathrm{Mpc}$, each with $256^{3}$ particles on a grid 256 cells on a side, solved using the code of Gnedin \& Hui (1998). The cosmological parameters we used are the same as used for the previous PT plots; for the transfer functions, we used CAMB. For the initial conditions (IC's) of the simulations, we used the 2LPT (2nd-order Lagrangian Perturbation Theory) code (Scoccimarro 1998), run to $z=49$. 2LPT IC's have reduced transients over IC's generated from the Zel'dovich (1970) approximation, and are also more accurate for higher-order statistics (see Crocce, Pueblas, \& Scoccimarro 2006, and references therein). We also ran 400 2LPT simulations to $z=0,100$ of which had the same random-number seeds as the PM simulations.

Figure 3 shows average power spectra from the various simulations. We also show the HALOFIT non-linear power spectrum (Smith et al. 2003). The average PM power spectrum departs clearly from HALOFIT at $k \sim 0.14 \mathrm{~h} \mathrm{Mpc}^{-1}$, so this is roughly the point to which we trust the PM simulations. The second wiggle at $k \sim 0.12$ is attenuated in the PM power spectra relative to HALOFIT, but HALOFIT was not developed with particular attention to BAO's, so the PM simulations may still be trustworthy there. The average 2LPT power spectrum exhibits a gradual attenuation in power starting at $k \sim 0.05 h \mathrm{Mpc}^{-1}$, but we still analyse the 2LPT simulations at higher $k$ for their high-signal-to-noise covariance matrix, keeping in mind this caveat. Scoccimarro (private communication) speculates that the deficit in power using 2LPT is from the large amount of shell-crossing at $z=0$, and that smoothly

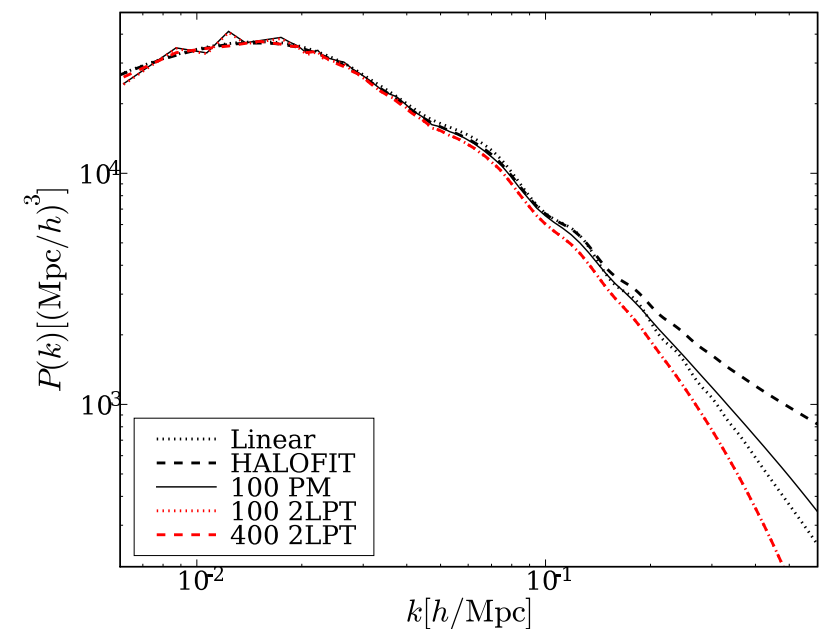

Figure 3. Power spectra of simulations used in the paper, averaged over the 100 or 400 realizations in each ensemble. The pure-2LPT simulations begin a gradual attenuation at rather large scales $\left(k \approx 0.05 h \mathrm{Mpc}^{-1}\right)$, while the particle mesh $(\mathrm{PM})$ simulations seem trustworthy to $k \approx 0.14 h \mathrm{Mpc}^{-1}$.

truncating the initial power for $k \gtrsim 0.3 h \mathrm{Mpc}^{-1}$ would improve 2LPT's performance.

To measure the power spectra, we used FFT's run on a $256^{3}$ mesh with cloud-in-cell density assignment. We corrected for the voxel window function by extrapolating the factor between power spectra measured from one of the simulations with $256^{3}$ and $1024^{3}$ grids. We subtracted off the Poisson shot noise of $64 h^{-3} \mathrm{Mpc}^{3}$, even though it is negligible over the range of scales we use.

Figure 4 shows measurements of $\tilde{T}(k)$ from a few ensembles of simulations. To measure $\tilde{T}$, we first measured power spectra in each simulation, using large bins to reduce noise. The bin edges were placed by hand to be approximately at the nodes between expected wiggles in $\tilde{T}$. The first bin runs from $0<k<$ $0.05 h \mathrm{Mpc}^{-1}$; the lowest $k$ sampled was $2 \pi / 1024 h^{-1} \mathrm{Mpc} \approx$ $0.006 h \mathrm{Mpc}^{-1}$. The edge of the first bin, at $0.05 h \mathrm{Mpc}^{-1}$, was chosen to be about where the wiggles cease being prominent in Fig. 1 When trying to detect the wiggles in $\tilde{T}$, there is a trade-off in choosing the edge of the first bin; with increasing $k$, the signal increases with the number of modes, but the wiggles dampen. It is possible that the wiggles could be more prominent with another choice of bin edge. Subsequent bin edges are shown in the plot, where horizontal error bars end. For each ensemble, we calculated a covariance matrix from the simulations' power spectra, and found $\tilde{T}(k)$ by dividing the covariance in each bin by $P\left(k_{1}\right)^{2} P(k)$, where $P$ is the power spectrum measured from the simulations, averaged in each bin and over all simulations.

The vertical error bars on the points at $k \sim 0.1 h \mathrm{Mpc}^{-1}$ were obtained by jackknife resampling. This method differs from spatial-jackknife resampling which is often used in estimating errors in large-scale structure; in spatial-jackknife resampling, errors are estimated by excising small spatial regions from simulations. In our case, we form jackknife samples by excluding entire simulations one-at-a-time from the sample. The error bar width on an $N$ simulation point is $\sqrt{N-1}$ times the standard deviation of measurements of $\tilde{T}\left(k=0.1 \mathrm{~h} \mathrm{Mpc}^{-1}\right)$ from $N$ covariance matrices, each formed by excluding one simulation at a time from the sample.

The outer points at $k \approx 0.07$ and $0.12 h \mathrm{Mpc}^{-1}$ appear with cones indicating the robustness of the peak at $k \sim 0.1 \mathrm{~h} \mathrm{Mpc}^{-1}$. We do not show these outer points with their own error bars be- 


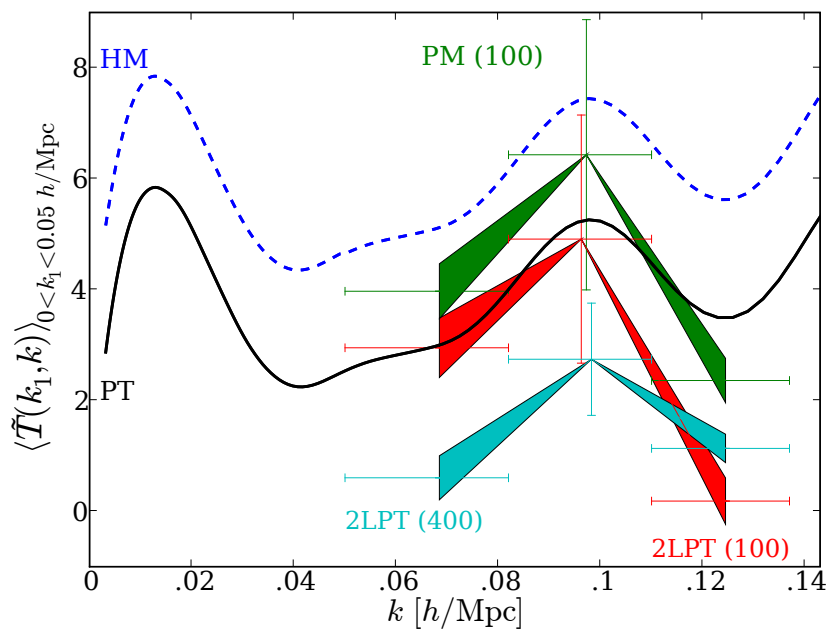

Figure 4. Measurements of the normalized power-spectrum covariance $\tilde{T}$ from ensembles of $N$-body simulations. $\tilde{T}$ is measured in large bins to beat down noise, with edges roughly where nodes in $\tilde{T}$ 's wiggles are expected. Only the middle points have error bars, which are typical of all three points, since different jackknife samples typically move up and down together. The cones coming from the middle points indicate the degree of robustness in the peak; see text for details. From 100 PM simulations of box size $1024 h^{-1} \mathrm{Gpc}$, we estimate a detection of the peak at a 5-sigma level. The simulations evolved entirely using 2LPT have somewhat lower values of $\tilde{T}$; the 100 2LPT simulations and the 100 PM simulations had the same initial conditions. With 300 additional 2LPT simulations, the noise in $\tilde{T}$ is reduced, and the peak shape is roughly that of the PT prediction.

cause there is a high degree of correlation among these three points. Typically, the curves for different jackknife subsamples just move up or down, and the peak-to-trough contrast hardly changes. The half-height of the cone at each $k_{i}$ is the standard deviation (times $\sqrt{N-1})$ of the differences $\left[\tilde{T}\left(0.1 h \mathrm{Mpc}^{-1}\right)-\tilde{T}\left(k_{i}\right)\right]$ measured from the $N$ jackknife measurements.

The data in Figure 4 do not indicate any extra contribution (such as the HM would give) to $\tilde{T}$ above the PT prediction. Both the PT and HM predictions are within the errors of the two leftmost points for the PM ensemble, and these points may be upward fluctuations in $\tilde{T}$ for the first 100 realizations. For the 2LPT ensemble, adding 300 more realizations to the sample moves the first two data points down, and the last point up. So, we recommend the simple PT covariance for the matter power spectrum for $k \lesssim$ $0.1 h \mathrm{Mpc}^{-1}$. However, the PT covariance must fail at some large $k$, so for analyses extending to larger $k$ than this, we would recommend using the halo-model covariance, excluding the offending $3 \mathrm{~h}$ term. This is rather arbitrary, but Neyrinck, Szapudi \& Rimes (2006, NSR) showed that the $1 \mathrm{~h}$ and (less so) the $2 \mathrm{~h}$ terms dominate the contribution to Fisher information loss in the halo-model matter power spectrum on non-linear scales, and thus the $1 \mathrm{~h}$ and $2 \mathrm{~h}$ terms are important to include. We do not exclude the $4 \mathrm{~h}$ term, since it is where the wiggles we are investigating lie.

Figure 5 shows $\tilde{T}$ measured with greater resolution. With these narrow bins, only for the 400-2LPT ensemble was the level of noise low enough to view calmly. The error bars are the square roots of the diagonal elements of the $\tilde{T}$ covariance matrix, again jackknife-estimated. To reduce the noise, we also measured $\tilde{T}$ using the weightings method of HRS. This gives 52 estimates of the power spectrum per simulation, by passing two differently phased long-wavelength sinusoidal window functions through each vertex, face, and edge of the simulation box. When excluding a simulation

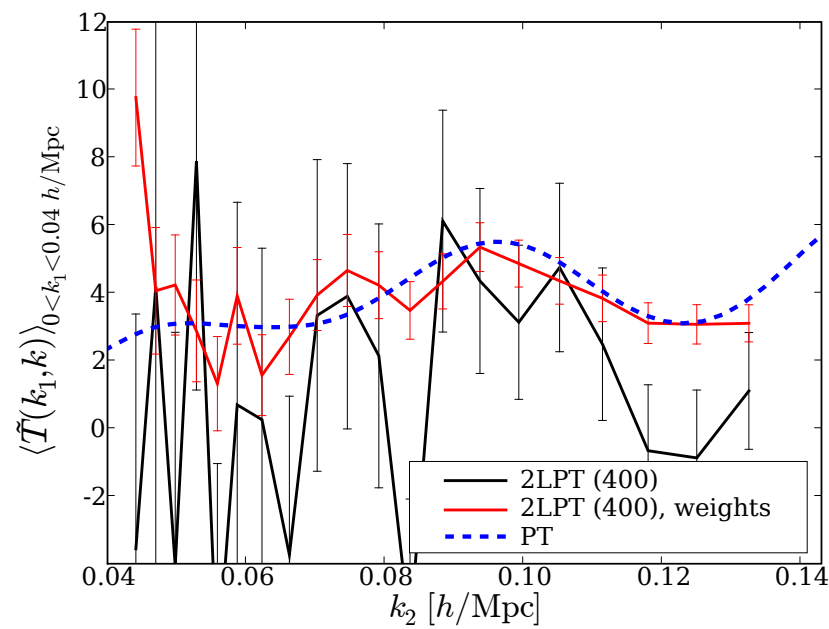

Figure 5. Higher-resolution (more-finely binned) measurements of the normalized power-spectrum covariance $\tilde{T}$ from 400 2LPT simulations. The red curve is $\tilde{T}$ measured using 52 weightings per simulation, according to the prescription of Hamilton, Rimes \& Scoccimarro (2006). The red curve is probably raised from the true level of $\tilde{T}$ in the 2LPT simulation because of the beat-coupling which the weightings induce.

from a jackknife sample using weightings, we excluded all of its 52 power spectra at a time.

The reduction in noise from the weightings method is substantial, and brings out the shape of $\tilde{T}$. However, we noticed in this and other investigations that the covariance estimated in this manner was a bit higher than the covariance from the unweighted simulations. This is likely a result of beat coupling to large scales that HRS discuss. Beat coupling refers to the extra covariance from non-trivial geometry (roughly, edge effects) in a power spectrum measured with finite bins. The weightings, even though they are minimally invasive, smear power among nearby wavenumbers as any survey window function does. When bins have a finite width, as they almost always do, this smearing contributes to the covariance a trispectrum term involving the power spectrum at the 'beat' wavenumber (the difference between the nearby wavenumbers). We did not use the weightings method for the investigations shown in Fig. 4 but a method such as the weightings method would probably be necessary to detect covariance-matrix wiggles in real surveys, and in doing this, beat-coupling should be taken into account. However, beat-coupling seems approximately to cause a wholesale additive shift in $\tilde{T}$, so its effects could be negligible if all one cares about is the position of wiggles in $\tilde{T}$. Because of beat coupling, we view it as a coincidence that $\tilde{T}$ from the 400 weighted 2LPT simulations lies right on the PT estimate; its true level for the 2LPT simulations is lower, as in Fig. 4 We suspect that the lower level of $\tilde{T}$ in the 2LPT ensemble is related to 2LPT's diminishing accuracy on small scales, as shown by the small-scale deficit in its power spectra, shown in Fig. 3

\subsection{Detectability of covariance-matrix wiggles}

We can also estimate roughly how big a survey is necessary to detect BAO's in $\tilde{T}$ from Fig. 4 In $100 \mathrm{PM}$ simulations $1024 \mathrm{~h} \mathrm{Mpc}^{-1}$ on a side (with a total volume of $107 h^{-3} \mathrm{Gpc}^{3}$ ), we detected the peak at a 5- $\sigma$ (4.97- $\sigma$, to be exact) level. This is the probability that $\tilde{T}(0.07)<\tilde{T}(0.1)$, as estimated by dividing the mean of $[\tilde{T}(0.1)-\tilde{T}(0.07)]$ by the standard deviation of this quan- 


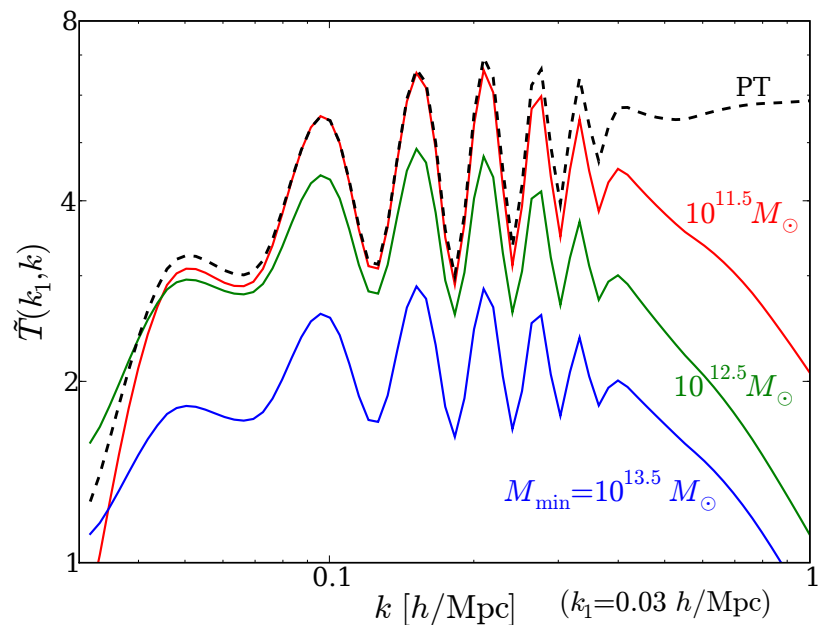

Figure 6. Wiggles in off-diagonal elements of the covariance matrix of halo-model galaxy power spectra, as shown for the matter power spectrum in Fig. 2 A few different Halo Occupation Distributions are shown, labeled by the minimum mass for a halo to host a central galaxy.

tity (the half-height of the cone). Doing the same on the right side of the peak gives an estimate of $10 \sigma$ for the probability that $\tilde{T}(0.1)>\tilde{T}(0.12)$.

We do not know precisely how the error bars in the difference between the peak and the troughs shrink with volume, but we can roughly estimate this by comparing the results from the ensembles of 100 and 400 2LPT simulations. There are two separate effects reducing the error bars: the increase in the number of power spectra used for the covariance matrix, as well as the increase in raw volume. The relationship between error-bar width and volume is not necessarily a simple power law. Still, it provides an easy estimate: this 'difference error bar' at $k \approx 0.7$ scales as $V^{0.22}$, and at $k \approx 0.12$ it scales as $V^{0.35}$. Taking the higher of these, an estimate of the volume of a survey that would give a 1- $\sigma$ detection of the peak is $107 / 5^{1 / 0.35} h^{-3} \mathrm{Gpc}^{3}=1.1 h^{-3} \mathrm{Gpc}^{3}$. Similarly, 2, 3 , and $4-\sigma$ detections could be done with 8,25 , and $57 h^{-3} \mathrm{Gpc}^{3}$ surveys, respectively. If we conservatively assume that error bars $\propto \sqrt{V}$ (as one might first guess), then we estimate that $1,2,3$, and $4-\sigma$ detections could be done with $4,17,39$, and $68 h^{-3} \mathrm{Gpc}^{3}$ surveys.

The wiggles in $\tilde{T}$ would almost certainly be measured with galaxies, not matter. Figure 6 shows $\tilde{T}$ of galaxies using the HM, using a few different halo occupation distributions (HOD's). These HOD's will be discussed in more detail in Sect. 4.1 but for now, note that the wiggles in $\tilde{T}$ for galaxies inhabiting smaller haloes are approximately like those for the matter, and the wiggles dampen somewhat with increasing host-halo mass. However, some of this dampening is done by the $3 \mathrm{~h}$ term, which apparently contributes artificially to the HM prediction in Fig. 2 So, there is some reason to doubt the dampening of the wiggles in $\tilde{T}$. The situation is a bit more complicated here than previously because we divide by $P_{\mathrm{g}}$, the galaxy power spectrum for each galaxy sample, instead of the linear power spectrum $P_{\text {lin }}$, to get $\tilde{T}\left(k_{1}, k\right)=$ $C\left(k_{1}, k\right) V /\left[P\left(k_{1}\right)^{2} P(k)\right]$.

Besides the differences in $\tilde{T}$ itself that galaxies would introduce, several factors would complicate detecting $\tilde{T}$ in reality. Redshift space distortions and complicated survey geometries would both need to be taken into account. As the discrepancy with the weightings method shows (Fig. 5], even a minimal window func- tion affects measured covariances. However, in that case, it seems that the window function roughly adds a simple constant to $\tilde{T}$, so it is possible that more complicated survey geometries would also roughly preserve the shapes of the wiggles.

There are also factors that would tend to increase detectability. First, as occurs exactly in PT, the galaxy $\tilde{T}$ is likely close to independent of redshift, removing one of the usual complications of measuring clustering statistics with a huge redshift survey. Also, we have not included the fact that a larger survey would allow largerscale modes $k_{1}$ to be accessed, where the wiggles in $\tilde{T}\left(k_{1}, k\right)$ are more pronounced. And, it is always possible that there exists another method than we have used to detect the wiggles more efficiently.

We have not explicitly investigated the issue of whether the wiggles in $\tilde{T}$ could help significantly in fixing the baryon acoustic scale, but it is worth further investigation, since every bit helps. One might first guess that it would be easier to measure BAO's in a three-point statistic than a four-point statistic such as $\tilde{T}$. However, the particular parallelogram configurations of the trispectrum which average to make $\tilde{T}$ depend only on the amplitude of each Fourier mode. Real numbers are not only easier to deal with numerically, but we conjecture that statistics independent of phases have smaller cosmic variance, since they are independent of phase correlations which increase for smaller survey volume. For these reasons, and for the simplicity of the algorithm of the estimator, these configurations of the trispectrum are competitive, perhaps even advantageous over using the bispectrum for detecting BAO's. Thus it would be interesting to confirm our predictions in data, since this would further corroborate our picture of gravitational structure formation, and, in particular, demonstrate that our understanding of BAO's extends to multipoint statistics.

\section{EFFECT ON COSMOLOGICAL PARAMETER ESTIMATION}

Baryon oscillations have become quite popular as a prospective cosmological probe. Here we consider to what degree BAO's in the covariance matrix affect these prospects, both for the matter power spectrum and galaxy power spectra. We investigate two parameters that directly involve BAO's: the baryon acoustic scale, and the baryon fraction $f_{b} \equiv \Omega_{b} /\left(\Omega_{b}+\Omega_{\mathrm{CDM}}\right)$. We do not investigate the baryon acoustic scale directly; we investigate the logarithm of the sound horizon when the baryons are released from the Compton drag of photons (see Eisenstein \& Hu 1998, EH). This is proportional to the baryon acoustic scale, i.e. the location of the peak in the linear correlation function.

We use a Fisher matrix formalism (Fisher 1935; Tegmark, Taylor \& Heavens 1997) to estimate error bars on these quantities, in the same way as in NS. The cumulative Fisher information in parameters $\alpha$ and $\beta$ over a range of bin indices $i \in \mathcal{R}$ is approximated (assuming a Gaussian likelihood function) as

$F_{\alpha \beta}(\mathcal{R})=\sum_{i, j \in \mathcal{R}} \frac{\partial \ln P_{i}}{\partial \alpha}\left(\mathbf{C}_{\mathcal{R}}^{-1}\right)_{i j} \frac{\partial \ln P_{j}}{\partial \beta}$,

where $\mathbf{C}_{\mathcal{R}}$ is the square submatrix of $\mathbf{C}$ with both indices ranging over $\mathcal{R}$. For simplicity, in this paper, we just consider singleparameter (unmarginalized) half-error bars; the half-error bar in $\alpha$ is $\sigma(\alpha) \equiv 1 / \sqrt{F_{\alpha \alpha}}$.

As recommended above in Sect. 3, we use the halo-model trispectrum, excluding the $3 \mathrm{~h}$ term, to compute the matter power 


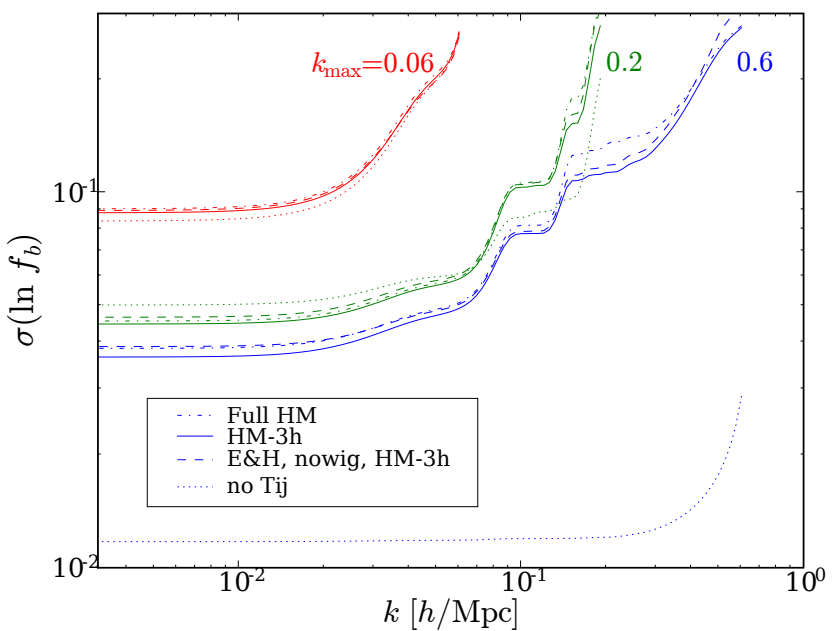

Figure 7. The effect of using different covariance matrices when analyzing matter power spectra to constrain the logarithm of the baryon fraction. Each curve starts at a $k_{\max }$, and shows how error bars tighten as larger scales are included in the analysis. The fiducial covariance matrix we recommend (the halo model covariance, minus the $3 \mathrm{~h}$ term) has been used for the solid curves. A no-wiggle power spectrum was used to produce the covariance matrices used for the dashed curves. Only the Gaussian variance is used for the dotted curves. The wiggles in the covariance matrix reduce $\sigma\left(\ln f_{b}\right)$ by $7 \%$ for $k_{\max }=0.6$.

spectrum covariance matrix. We do, however, show the results when the full $\mathrm{HM}$ covariance (including the $3 \mathrm{~h}$ term) is included; it does not make a big difference. We do not use simply the PT covariance because we investigate well into the non-linear regime, up to almost $k=1 \mathrm{~h} \mathrm{Mpc}^{-1}$.

Figure 7 shows what effect $T_{i j}$ terms in the covariance matrix have on estimation of the baryon fraction from the matter power spectrum. For this and subsequent figures, we assume a fixed volume of $1 h^{-3} \mathrm{Gpc}^{3}$. Each curve starts on the right at a $k_{\max }$, and shows how error bars in $\ln f_{b}$ tighten as the power spectrum is measured over an increasing range of $k$. Four curves depart from each $k_{\max }$ curve, calculated using different covariance matrices: a purely Gaussian covariance matrix, including just the $P(k)^{2}$ term on the diagonal; a 'HM-3h' covariance matrix, our fiducial covariance matrix, which uses the full HM except for the 3-halo term; a full halo-model covariance matrix; and a 'HM-3h, nowig' covariance matrix, which uses a no-wiggle transfer function of $\mathrm{EH}$ instead of CAMB to obtain the input power spectrum. We normalize the 'nowig' power spectrum to match the CAMB power spectrum in amplitude at $k=10^{-5} h \mathrm{Mpc}^{-1}$, and also set it equal to the CAMB power spectrum for $k>130 h \mathrm{Mpc}^{-1}$, the wavenumber where the two power spectra cross on scales smaller than the BAO regime. Fig.9 (mainly about galaxy power spectra, discussed in Sect. 4.1) shows the halo-model matter power spectrum, along with its no-wiggle counterpart.

Going well into the non-linear regime (looking at the blue curve, with $k_{\max }=0.6 \mathrm{~h} \mathrm{Mpc}^{-1}$ ), the wiggly covariance matrix gives about $7 \%$ tighter error bars, going to small $k$, than the non-wiggly covariance matrix. The difference is also $7 \%$ if a full HM covariance matrix is used for each. The purely Gaussian covariance matrix clearly gives underestimated error bars here. This is because of the 'translinear information plateau;' significant correlations arise from halo mass-function fluctuations between

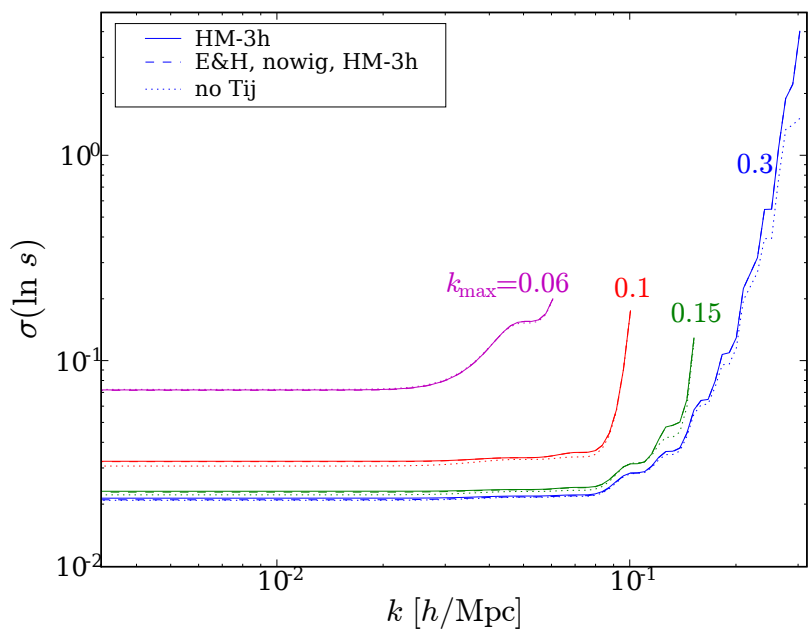

Figure 8. As in Fig. 7 except showing error bars on the baryon acoustic scale (actually, the logarithm of the sound-horizon scale). Here, the nonGaussian covariance matrix terms are essentially negligible. The wiggles in the covariance matrix enlarge $\sigma\left(\ln f_{b}\right)$ by $2 \%$ for $k_{\max }=0.3$.

power-spectrum bins in the range $0.2 \lesssim k /\left(h \mathrm{Mpc}^{-1}\right) \lesssim 0.8$ (Rimes \& Hamilton 2005, 2006, NSR, NS).

However, in an intermediate regime (looking at the green curve, with $k_{\max }=0.2 \mathrm{~h} \mathrm{Mpc}^{-1}$ ), the full covariance matrix actually gives tighter error bars than if just the Gaussian term is used. Here, the wiggly covariance matrix gives tighter error bars by $4 \%$ than the non-wiggly one. Starting on larger scales (looking at the red curve, with $k_{\max }=0.06 h \mathrm{Mpc}^{-1}$ ), the covariance matrices including $T_{i j}$ terms again give somewhat larger error bars than if only the Gaussian term is used.

The derivative terms $\partial \ln P(k) / \partial \ln f_{b}$ are the same for all calculations in Fig. 7 For this, we calculated linear power spectra with CAMB using slightly different baryon fractions, at fixed $\sigma_{8}$. We then put these varied linear power spectra through our halomodel code. The rise of the one-halo term on small scales does attenuate the BAO's somewhat compared to the linear power spectrum, but not to the degree seen in $N$-body simulations. We thus used the method described by Percival et al. (2007b, P07), based on the work of Eisenstein et al. (2007). The wiggles are attenuated by Gaussian window in Fourier space of width $1 /\left(10 h^{-1} \mathrm{Mpc}\right)$.

Figure 8 is the same as Fig. 7 except it shows error-bar tightening in a quantity more popular in BAO parameter estimation, the baryon acoustic scale (we actually show error bars in the logarithm of the sound horizon). In this case, the error bars using the wiggly covariance matrix differ nearly indistinguishably from those using the non-wiggly ones; going from arbitrarily large scales to $k_{\max }=0.3$, there is only a $2 \%$ difference in the error bars. In fact, excluding all $T_{i j}$ terms makes at most a $6 \%$ difference in the error bars. The 'Full HM' curves have been left off this plot, since they are indistinguishable from the 'HM- $3 \mathrm{~h}$ ' curves.

In Fig. 8, again we use the same derivative terms $\partial \ln P(k) / \partial \ln s$ for each curve. We estimate $\partial \ln P(k) / \partial \ln s$ by changing the sound horizon by hand in the transfer function code of EH. This is not terribly physical, but it does what we want: changing $s$ by hand in the EH code results in power spectra which are effectively identical, except that the BAO's are offset. As with our investigation of the baryon fraction, we attenuate the baryon wiggles with a Gaussian window.

It is interesting that the wiggles in the covariance matrix gen- 
erally result in somewhat narrower constraints in $\ln f_{b}$. Intuitively, it makes sense that knowledge of how BAO's are attenuated by fluctuations in large-scale power would help to constrain $f_{b}$, which directly affects the BAO amplitude.

The effect of $\tilde{T}$ is smaller for sound-horizon determination. Numerically, this is for two reasons: one, the derivative term $\partial \ln P(k) / \partial \alpha$ is effectively zero on large scales. Also, the wiggles in the derivative term are well out-of-phase with wiggles in the Fisher matrix, $\mathbf{C}^{-1}$. The difference might be greater if the BAO's in $\tilde{T}$ were not so close to entirely out-of-phase with the BAO's in the power spectrum; that is, $\tilde{T}$ might have a greater effect if large-scale power appreciably moved the BAO's.

\subsection{Wiggles in galaxy power spectra}

In the previous section, we showed that BAO's in the covariance matrix of the matter power spectrum have a several-percent effect on the inferred error bars on the baryon fraction $f_{b}$, and a smaller effect on error bars in the sound horizon $s$. Here we investigate to what degree these effects propagate through to galaxy power spectra.

We use a halo occupation distribution (HOD, e.g. Berlind \& Weinberg 2002) to model the galaxy power spectrum and covariance matrix. We build off of the matter trispectrum as worked out by $\mathrm{CH}$, and use the satellite HOD as introduced by Kravtsov et al. (2004, K04), with its simple Poisson satellite HOD moments. See Appendix B for details of our implementation.

In the K04 model, a halo has a central galaxy if and only if the halo has a dark-matter mass $m>M_{\min }$. The other two parameters in the HOD are $M_{1}$, the mass at which, on average, a halo has one satellite galaxy, and $\gamma$, the slope in the formula giving the mean number of satellites in a halo as a function of mass;

$\left\langle N_{s} \mid m\right\rangle=\left(m / M_{1}\right)^{\gamma}$.

Three parameters give a huge potential parameter space to explore. To narrow it, we vary $M_{\min }$, and fix $M_{1} / M_{\min }=30$ (which K04 suggested as a fiducial value). Observed galaxy power spectra at $z=0$ are generally close to power laws, so to use the most physically plausible HOD's, we set $\gamma$ at each $M_{\text {min }}$ by maximizing the galaxy power spectrum's straightness. More precisely, we minimized the sum of the squares of the second derivative of $P_{\mathrm{g}}(k)$ in $\log$-log space over a range $10^{-0.5}<k /\left(h \mathrm{Mpc}^{-1}\right)<100$. The three halo masses $10^{11.5}, 10^{12.5}$, and $10^{13.5} M_{\odot}$ have best-fitting values $\gamma=0.97,1.23$ and 1.68 .

To account for galaxy shot noise, we use the shot-noise-added power spectrum $\left(P_{\mathrm{g}}(k)+1 / \bar{n}_{\mathrm{g}}\right)$ for the Gaussian terms on the diagonal of the covariance matrix (e.g. Cooray 2004). Here, $\bar{n}_{\mathrm{g}}$ is the number density of galaxies. This treatment of shot noise is equivalent to using an 'effective volume' $V^{\text {eff }}(k)=V /\{1+$ $\left.1 /\left[\bar{n}_{\mathrm{g}} P_{\mathrm{g}}(k)\right]\right\}^{2}$ at each $k$ (e.g. Tegmark et al. 1997). The shot noises for the three HOD's used, in increasing order of $M_{\min }$, are $1 / \bar{n}=6200,540$, and $60 \mathrm{~h} \mathrm{Mpc}^{-3}$. For the smallest- $M_{\min }$ sample, the shot noise is negligible up to $k=1 \mathrm{~h} \mathrm{Mpc}^{-1}$; for the two

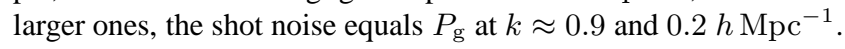

Figure 9 shows the power spectra for the HOD's we investigate below, along with the matter power spectrum investigated in Sect. 4 the CAMB linear power spectrum, and the HALOFIT non-linear power spectrum. We also show each power spectrum as produced from the EH no-wiggle power spectrum. Each power spectrum is divided by the EH no-wiggle linear power spectrum for clarity. The wiggles in the matter and galaxy power spectra here are only attenuated by the rising, unwiggly $1 \mathrm{~h}$ term. The only

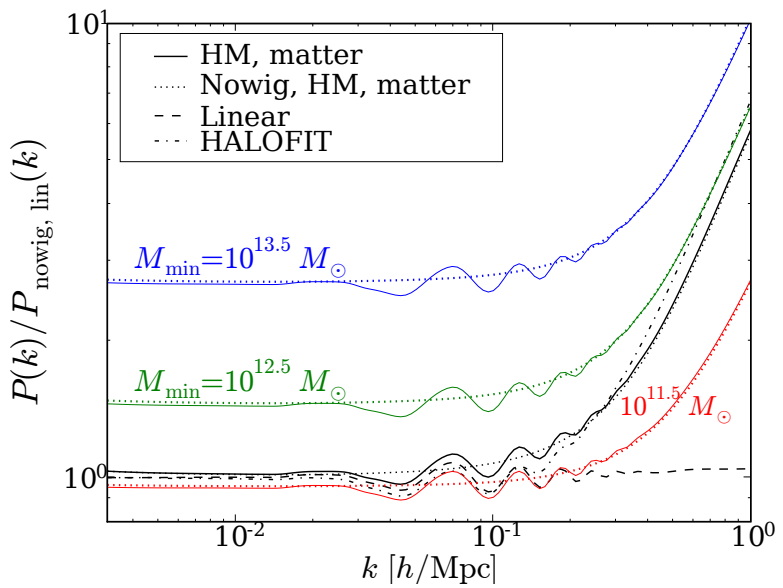

Figure 9. Various halo-model power spectra used in the paper, divided by an Eisenstein \& Hu (1998) no-wiggle power spectrum. The colored curves are halo-model galaxy power spectra, labeled by the minimum mass of a halo which hosts a central galaxy.

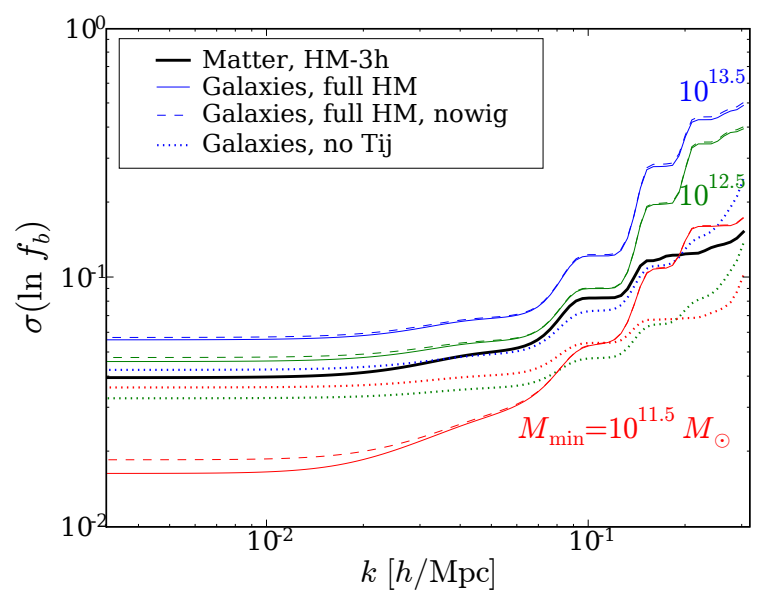

Figure 10. At fixed volume, error-bar widths on the logarithm of the baryon fraction $f_{b}$ from analyzing power spectra of a few different galaxy samples. This is as in Fig.7 which shows error-bars for matter power spectra, except here, $k_{\max }$ is fixed at $0.3 \mathrm{~h} \mathrm{Mpc}^{-1}$. The wiggles in the covariance matrix for galaxies have about the same influence on the error-bars on $\ln f_{b}$ as for matter, if not a bit more. Also, a significant reduction in error-bar width occurs for low $M_{\min }$ (the minimum halo mass which can support a central galaxy).

place where we implement the more-accurate method of P07 is in the derivatives of the power spectrum with respect to parameters, which is where it matters significantly for parameter estimation. We use the same P07 BAO attenuation for all galaxy and matter power spectra, since the attenuation in this model is a function of a single parameter, the width of a Gaussian window. It is unclear how to change this parameter for different galaxy populations.

Figures 10 and 11 show error-bar tightening in the baryon fraction and the sound horizon, in a similar manner as in Figs. 7 and 8 Here, we fix $k_{\max }=0.3 \mathrm{~h} \mathrm{Mpc}^{-1}$, but show results for three different $M_{\min }$ 's. We include the $3 \mathrm{~h}$ term in the galaxy co- 


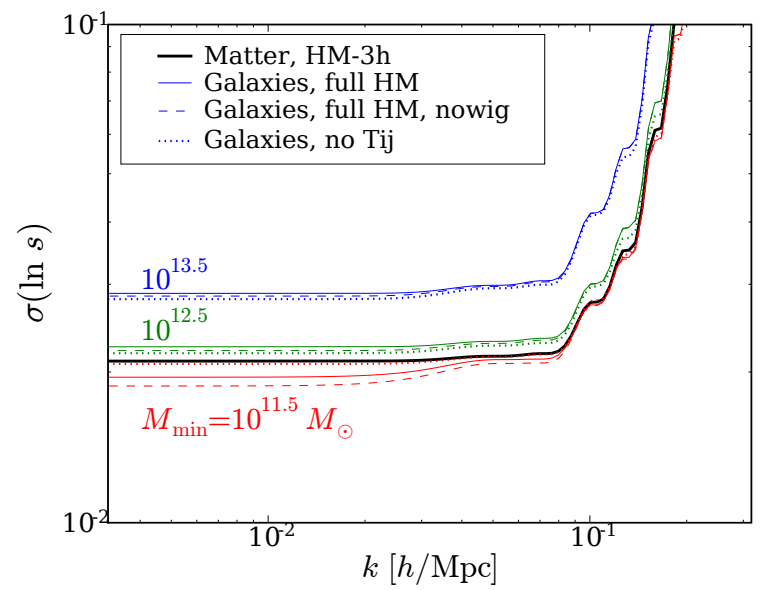

Figure 11. At fixed volume, error-bar widths on the logarithm of the sound horizon $s$ from analyzing power spectra of a few different galaxy samples. The wiggly covariance matrix here has a bit greater effect for galaxies than for matter; for comparison, see Fig. 8 but notice that the $y$-axis is inflated here relative to that figure.

variance matrix since we do not have theoretical arguments and measurements (as we do for matter) telling us to discard it.

At our fixed volume $V=1 h^{-3} \mathrm{Gpc}^{3}$, constraints are tighter for galaxies with a lower $M_{\min }$. There are two causes for this. First, galaxies with high $M_{\min }$ are sparser and therefore have more shot noise, an effect which wins out over their increase in bias. The shotnoise effect can roughly be judged by looking at the dotted curves at different $M_{\min }$ 's. The difference between the brightest and intermediate HOD's is mainly from this.

Second, the dominance of lower-halo (1h, $2 \mathrm{~h}$, and $3 \mathrm{~h}$ ) terms grows with $M_{\min }$ in the HM covariance matrix. This is what causes the drastic difference between the middle and faintest samples. It also increases the impact of covariance-matrix wiggles for the faintest galaxies; for this sample, the difference in error-bar width between the wiggly and non-wiggly covariance matrices reaches $13 \%$ for $\ln f_{b}$, and $4 \%$ for $\ln s$. The lower-halo terms attenuate the wiggles in $\tilde{T}$ increasingly for brighter galaxy samples, as shown in Fig.6

As $M_{\min }$ increases, not only are the effects of lower-halo terms greater on large scales, but the scale increases (i.e. the critical $k$ reduces) where lower-halo terms come to entirely dominate the covariance. Thus, if $M_{\min }$ is small, the wiggles in the covariance matrix can be accessed over a larger range of scales. Roughly, if the $1 \mathrm{~h}$ term comes in on smaller scales in the power spectrum (as it does for the fainter galaxy samples), then more cosmological information can be measured from the $2 \mathrm{~h}$ (quasi-linear) term.

This is similar to what we found in NS; cosmological information is more pristine in regions of the universe in earlier stages of structure formation. It seems likely that, again as in NS, imposing an $M_{\max }$, i.e. excluding galaxies in large clusters, could help significantly to tap smaller-scale information. However, just as in that study, we find that our model breaks down when the lowest-mass haloes dominate the power spectrum and its covariance matrix. For $M_{\text {min }} \lesssim 10^{11} M_{\odot}$, using values of $\gamma$ fit in the same way as for the samples we display, we sometimes get non-positive-definite covariance matrices. (However, it is possible with slightly different $\gamma$ 's to get well-behaved covariance matrices.)

We attribute this breakdown for small $M_{\min }$ to our lack of accurate knowledge about the power spectrum and trispectrum of haloes $\left(P_{\mathrm{hh}}\right.$ and $\left.T_{\mathrm{hhhh}}\right)$ in the translinear regime. Smith, Scoccimarro \& Sheth (2007a) showed that $P_{\text {hh }}$ is difficult to model; $T_{\mathrm{hhhh}}$ is likely harder. Our assumption that leading-order PT describes the polyspectra of haloes is not unreasonable, but the leading order is different for each polyspectrum. Using different orders of PT for the power spectrum and trispectrum together is inconsistent in the translinear regime, where higher-order corrections to the power spectrum are significant. For the matter power spectrum, and for high $M_{\min }$, the $1 \mathrm{~h}$ terms of both the power spectrum, and its covariance, dominate terms involving $P_{\mathrm{hh}}$ and $T_{\mathrm{hhhh}}$ on translinear scales. In these cases, accurate modelling of $P_{\mathrm{hh}}$ and $T_{\text {hhhh }}$ would make a negligible difference in our calculations. When the $1 \mathrm{~h}$ terms are reduced relative to other terms, though, the raw $P_{\mathrm{hh}}$ and $T_{\mathrm{hhhh}}$ are exposed in a regime where they are not known accurately.

Because the $M_{\min }=10^{11.5} M_{\odot}$ sample is close to the critical $M_{\text {min }}=10^{11} M_{\odot}$ where covariance matrices can be non-positivedefinite for fiducial $\gamma$ 's and $M_{1}$ 's, we view the drastic diminution in the error bars using this sample with some caution. Still, we confidently assert that in the HM, reducing $M_{\text {min }}$ can produce significantly smaller error bars than the simple reduction in shot noise would suggest.

Even though there seem to be significant gains in cosmological information when using a fainter galaxy sample, it is important to remember that we are holding the volume of the survey fixed. We have not considered some important realistic effects, an obvious one being that it takes less telescope time to measure bright than faint galaxies.

The question of how to choose a galaxy sample to maximize cosmological information is a very important and interesting one, but it lies outside the scope of this paper. The answer to the main question of this section is this: wiggles in galaxy power-spectrum covariance matrices do seem to affect constraints on $\ln f_{b}$ and $\ln s$ to about the same (if not somewhat larger) degree as for matter.

\section{CONCLUSIONS}

Our main points are the following:

- In off-diagonal entries in power spectrum covariance matrices, BAO's exist which appear much stronger than the BAO's in the power spectrum. These wiggles are a manifestation of the suppression which large-scale power does to BAO's in the power spectrum, and originate in the perturbation-theory trispectrum. We give a simple analytic approximation to these wiggles in terms of the linear power spectrum and its first two derivatives, and check the analytical predictions using $N$-body simulations.

- These wiggles are potentially detectable in current and upcoming surveys, but because of the large noise in a covariance matrix measurement, they are only detectable at a small significance level. We estimate that a one-sigma detection could be done with a survey of size a couple of $h^{-3} \mathrm{Gpc}^{3}$, and that a three-sigma detection could require a survey of volume $\sim 30 h^{-3} \mathrm{Gpc}^{3}$.

- The wiggles make a modest difference in cosmological parameter error bars from analysing galaxy and matter power spectra. For example, using the true, wiggly covariance matrix in estimating the baryon fraction results in error bars several percent tighter than a no-wiggle covariance matrix. Doing so in estimating the baryon acoustic scale has a smaller effect.

- At fixed volume, including galaxies in smaller-mass haloes 
provides tighter error bars on parameters such as the baryon fraction and the baryon acoustic scale than analysing galaxies in only large haloes. In the context of the HM, this effect goes beyond the simple gains from analysing a sample with smaller shot noise. We attribute this to the one-halo term of the galaxy power spectrum becoming dominant at smaller scales for small haloes than large ones.

Most of the calculations in this paper made use of our package of Python code for cosmology, called CosMOPY. It can be downloaded from http: //www.ifa.hawaii.edu/cosmopy/

\section{ACKNOWLEDGMENTS}

We thank Adrian Pope, Ben Granett, Martin White, Alex Szalay and Tom Bethell for helpful discussions, Nick Gnedin for access to his PM code, and an anonymous referee for perspicacious questions and comments. We are grateful for support from NASA grant NNG06GE71G, and NSF grants AST-0206243, ITR 1120201-128440, and AMS04-0434413.

\section{REFERENCES}

Angulo R.E., Baugh C.M., Frenk C.S., Lacey C.G., 2007, MNRAS submitted, astro-ph/0702543

Berlind A.A., Weinberg D.H., 2002, ApJ, 575, 587

Bernardeau F., Colombi S., Gaztañaga E., Scoccimarro R., 2002, Phys. Rep., 367, 1

Bouchet F.R., Juszkiewicz R., Colombi S., Pellat R., 1992, ApJ, 394, L5

Blake C., Glazebrook K., 2003, ApJ, 592, 665

Bullock J.S., Kolatt T.S., Sigad Y., Somerville R.S., Kravtsov A.V., Klypin A.A., Primack J.R., Dekel, A., 2001, MNRAS, 321, 559

Cole S., et al., 2005, MNRAS, 362, 505

Cooray A., 2004, MNRAS, 348, 250

Cooray A., Hu W., 2001, ApJ, 554, 56 (CH)

Cooray A., Sheth R., 2002, Phys. Rep., 372, 1

Crocce M., Scoccimarro R., 2007, MNRAS, submitted, astro$\mathrm{ph} / 0704.2783$

Crocce M., Pueblas M., Scoccimarro R., 2006, MNRAS, 373, 369

Eisenstein D.J., Hu W., ApJ, 496, 605 (EH)

Eisenstein D.J., et al., 2005, ApJ, 633, 560

Eisenstein D.J., Seo H.-J., White M., 2007, ApJ, 664, 660

Fisher R.A., 1935, J.Roy.Stat.Soc., 98, 39

Goroff M.H., Grinstein B., Rey S.-J., Wise M.B., 1986, ApJ, 311, 6

Gnedin, N.Y., Hui L., 1998, MNRAS, 296, 44

Hamilton A.J.S., Rimes C.D., Scoccimarro R., 2006, MNRAS, 371, 1188 (HRS)

Holtzman J.A., 1989, ApJS, 71, 1

Huff E., Schulz A.E., White M., Schlegel D.J., Warren M.S., 2007, Astroparticle Physics, 26, 351

Hütsi G., 2006, A\&A, 449, 891

Jeong D., Komatsu E., 2006, ApJ, 651, 619

Lewis A., Challinor A., Lasenby A., 2000, ApJ, 538, 473

Kravtsov A.V., Berlind A.A., Wechsler R.H., Klypin A.A., Gottlöber S., Allgood B., Primack J.R., 2004, ApJ, 609 (K04)

Matsubara T., 2007, preprint (arXiv:0711.2521)

McDonald P., 2007, Phys. Rev. D 75, 043514

Meiksin A., White M., Peacock J.A., 1999, MNRAS, 304, 851
Mo H.J., Jing Y.P., White S.D.M., 1997, MNRAS, 284, 189

Nagai D., Kravtsov A.V., 2005, ApJ, 618, 557

Navarro J., Frenk C., White S.D.M., 1996, ApJ, 462, 563

Neyrinck M.C., Szapudi I., 2007, MNRAS, 375, L51 (NS)

Neyrinck M.C., Szapudi I., Rimes C.D., 2006, MNRAS, 370, L66 (NSR)

Peebles P.J.E., Yu J.T., 1970, ApJ, 162, 815

Percival W.J., et al., 2007a, ApJ, 657, 645

Percival W.J., Cole S., Eisenstein D.J., Nichol R.C., Peacock J.A., Pope A.C., Szalay A.S., 2007b, MNRAS, 381, 1053 (P07)

Rimes C.D., Hamilton A.J.S., 2005, MNRAS, 360, L82

Rimes C.D., Hamilton A.J.S., 2006, MNRAS, 371, 1205

Scoccimarro R., 1998, MNRAS, 299,1097

Scoccimarro R., Sheth R., Hui L., Jain B., 2001, ApJ, 546, 20 (SSHJ)

Scoccimarro R., Zaldarriaga M., Hui L., 1999, ApJ, 527, 1

Seo H.-J., Eisenstein D.J., 2003, ApJ, 598, 720

Seo H.-J., Eisenstein D.J., 2007, ApJ, 665, 14

Sheth R.K., Tormen G., 1999, MNRAS, 308, 119

Schulz A. E., White M., 2006, Astroparticle Physics, 25, 172

Smith R.E., Scoccimarro R., Sheth R.K., 2007a, Phys. Rev. D, 75, 063512

Smith R.E., Scoccimarro R., Sheth R.K., 2007b, Phys. Rev. D submitted, astro-ph/0703620

Smith R.E., Watts P.I.R., Sheth R.K., 2006, MNRAS, 365, 214

Smith R.E. et al., 2003, MNRAS, 341, 1311

Springel V., et al., 2005, Nature, 435, 629

Sunyaev R.A., Zel'dovich Ya.B., 1970, Ap\&SS, 7, 3

Tegmark M., 1997, Phys. Rev. Lett., 79, 3806

Tegmark M., Taylor A.N., Heavens A.F., 1997, ApJ, 480, 22

Valageas P., 2004, A\&A, 421, 23

Zel'dovich Ya.B., 1970, A\&A, 5, 84 


\section{APPENDIX A: WIGGLES IN THE PERTURBATION-THEORY TRISPECTRUM}

The expression for the 3rd-order PT trispectrum includes 12 terms involving the $F_{2}$ symmetrized kernel, and 4 terms involving the $F_{3}$ symmetrized kernel. In this Appendix, all appearances of $F_{2}$ and $F_{3}$ are the symmetrized kernels, often denoted in the literature with a superscript ${ }^{(s)}$. Expressions for these mode-coupling kernels can be found in, for example, Goroff et al. (1986) and Bernardeau et al. (2002). The $F_{2}$ kernel is

$F_{2}\left(\boldsymbol{k}_{1}, \boldsymbol{k}_{2}\right)=\frac{1}{2}\left[1+\mu+\left(\frac{k_{1}}{k_{2}}+\frac{k_{2}}{k_{1}}\right) c+(1-\mu) c^{2}\right]$,

where $c \equiv \cos \theta_{12}$. In the following, we take the Einstein-de Sitter value of $\mu=3 / 7$, even though it has a mild dependence on $\Omega_{m}$; $\mu \approx \frac{3}{7} \Omega_{m}^{-2 / 63}$ (Bouchet et al. 1992). This quantity $\mu$ also appears in the $F_{3}$ kernel. For calculations, we do use this expression for $\mu$; for our assumed cosmology, $\mu \approx 1.045 \times \frac{3}{7}$. The PT trispectrum is

$T\left(\boldsymbol{k}_{1}, \boldsymbol{k}_{2}, \boldsymbol{k}_{3}, \boldsymbol{k}_{4}\right)=4\left[F_{2}\left(\boldsymbol{k}_{13},-\boldsymbol{k}_{3}\right) F_{2}\left(\boldsymbol{k}_{24},-\boldsymbol{k}_{4}\right) P_{3} P_{4} P_{13}+\right.$ cyc. $]+6\left[F_{3}\left(\boldsymbol{k}_{2}, \boldsymbol{k}_{3}, \boldsymbol{k}_{4}\right) P_{2} P_{3} P_{4}+\right.$ cyc. $]$.

Here, $k_{13}=k_{1}+k_{3}$ and $P_{13}=P\left(k_{13}\right)$; in this Appendix, $P$ denotes $P_{\text {lin }}$, the linear power spectrum.

The abbreviation 'cyc.' describing the $12 F_{2}$ terms could do with some further explanation. The $F_{2}$ terms come from terms such as $\left\langle\delta^{(2)}\left(\boldsymbol{k}_{1}\right) \delta^{(2)}\left(\boldsymbol{k}_{2}\right) \delta^{(1)}\left(\boldsymbol{k}_{3}\right) \delta^{(1)}\left(\boldsymbol{k}_{4}\right)\right\rangle_{\mathrm{c}}$, where the superscript on $\delta$ is the order of perturbation. There are six choices of where to put the ${ }^{(2)}$ labels. Each of these gives two $F_{2}$ terms;

$\left\langle\delta^{(2)}\left(\boldsymbol{k}_{1}\right) \delta^{(2)}\left(\boldsymbol{k}_{2}\right) \delta^{(1)}\left(\boldsymbol{k}_{3}\right) \delta^{(1)}\left(\boldsymbol{k}_{4}\right)\right\rangle_{\mathrm{c}}=F_{2}\left(\boldsymbol{k}_{13},-\boldsymbol{k}_{3}\right) F_{2}\left(\boldsymbol{k}_{24},-\boldsymbol{k}_{4}\right) P_{3} P_{4} P_{13}+F_{2}\left(\boldsymbol{k}_{14},-\boldsymbol{k}_{4}\right) F_{2}\left(\boldsymbol{k}_{23},-\boldsymbol{k}_{3}\right) P_{3} P_{4} P_{14}$.

The PT trispectrum contribution to the power-spectrum covariance can be simplified to the following, given by Scoccimarro, Zaldarriaga \& Hui (1999) (which we rearrange):

$T_{i j}=\int_{k_{i}} \int_{k_{j}}\left\{12 P_{1} P_{2}\left[F_{3}\left(\boldsymbol{k}_{1},-\boldsymbol{k}_{1}, \boldsymbol{k}_{2}\right) P_{1}+F_{3}\left(\boldsymbol{k}_{2},-\boldsymbol{k}_{2}, \boldsymbol{k}_{1}\right) P_{2}\right]+8 P_{1-2}\left[F_{2}\left(\boldsymbol{k}_{2-1}, \boldsymbol{k}_{1}\right) P_{1}+F_{2}\left(\boldsymbol{k}_{1-2}, \boldsymbol{k}_{2}\right) P_{2}\right]^{2}\right\} \frac{d^{3} \boldsymbol{k}_{1}}{V_{s}\left(k_{i}\right)} \frac{d^{3} \boldsymbol{k}_{2}}{V_{s}\left(k_{j}\right)}$.

Here, $k_{1-2}=k_{1}-k_{2}$, and $P_{1-2}=P\left(\left|k_{1-2}\right|\right)$.

A possibly useful intermediate result for the $F_{2}$ part is, again where $c=\cos \theta_{12}$,

$F_{2}\left(\boldsymbol{k}_{2-1}, \boldsymbol{k}_{1}\right) P_{1}+F_{2}\left(\boldsymbol{k}_{1-2}, \boldsymbol{k}_{2}\right) P_{2}=\frac{\left[\left(3-10 c^{2}\right) k_{1}+7 c k_{2}\right] k_{2}^{3} P_{1}+\left(k_{1} \leftrightarrow k_{2}\right)}{14 k_{1} k_{2}\left(k_{1}^{2}+k_{2}^{2}-2 c k_{1} k_{2}\right)}$.

Where this time $c=\cos \theta_{a b}$, the $F_{3}$ part can be expressed as (Valageas 2004):

$F_{3}(\boldsymbol{a},-\boldsymbol{a}, \boldsymbol{b})=\frac{b^{2}\left[a^{4}\left(10-59 c^{2}+28 c^{4}\right)+a^{2} b^{2}\left(10-44 c^{2}+76 c^{4}\right)-21 b^{4} c^{2}\right]}{126 a^{2}\left[a^{4}+b^{4}+2 a^{2} b^{2}\left(1-2 c^{2}\right)\right]}$.

The main approximation we use is $k_{i} \ll k_{j}$; we keep terms up to zeroth order in $k_{1}$ (terms in $k_{2} / k_{1}$ to the powers 2 , 1, and 0 ). Substituting $P\left(k_{2}\right)-\left(c k_{1}\right) P^{\prime}\left(k_{2}\right)+\frac{1}{2}\left(c k_{1}\right)^{2} P^{\prime \prime}\left(k_{2}\right)$ for $P_{1-2}$ and averaging over $c$ gives

$T_{i j}=\left[\frac{5038}{2205} P\left(k_{j}\right)-\frac{36}{35} P^{\prime}\left(k_{j}\right) k_{j}+\frac{1}{5} P^{\prime \prime}\left(k_{j}\right) k_{j}^{2}\right] P\left(k_{i}\right)^{2}$,

which is the result in Eq. (3). There is no term involving a higher derivative of $P(k)$ to this order in $k_{i}$. Another approximation which makes a term involving $P\left(k_{i}\right) P\left(k_{j}\right)^{2}$ disappear in Eq. A7) is $\frac{k_{i}^{2}}{P\left(k_{i}\right)} \ll \frac{k_{j}^{2}}{P\left(k_{j}\right)}$.

\section{APPENDIX B: THE HALO-MODEL GALAXY TRISPECTRUM}

The galaxy trispectrum, excluding the effects of shot noise, has a similar form to the matter trispectrum, which $\mathrm{CH}$ worked out. The main difference is that matter densities get replaced by galaxy number densities.

In the HM, galaxies and dark matter are assumed to lie entirely within haloes. Like $\mathrm{CH}$, we assume that the haloes are distributed according to leading-order perturbation theory (PT), i.e. the linear power spectrum, and the 3rd-order PT trispectrum. This is not quite true (Smith, Scoccimarro \& Sheth 2007a), but there seems currently to be no better analytic approximation to use.

The matter power spectrum in the HM is the sum of two terms:

$P_{\mathrm{mm}}(k)=P_{\mathrm{mm}}^{1 \mathrm{~h}}(k)+P_{\mathrm{mm}}^{2 \mathrm{~h}}(k)=M_{2}^{0}(k, k)+P^{\operatorname{lin}}(k)\left[M_{1}^{1}(k)\right]^{2}$,

where $M_{\alpha}^{\beta}$ are integrals over the halo mass function $n(m)$.

$M_{\alpha}^{\beta}\left(k_{1}, \ldots, k_{\alpha}\right) \equiv \int_{0}^{\infty}(m / \bar{\rho})^{\alpha} u\left(k_{1}, m\right) \cdots u\left(k_{\alpha}, m\right) b_{\beta}(m) n(m) d m$.

Here, $\bar{\rho}$ is the mean matter density, $b_{\beta}(m)$ is the $\beta$-order halo bias (Mo, Jing \& White 1997; Scoccimarro et al. 2001, SSHJ), and $u(k, m)$ is the Fourier-transformed halo matter-density profile, for which we take a Navarro, Frenk \& White (1996) form. In principle, $u$ could be complex, but we assume a spherically symmetric form, which forces it to be real. We use a Sheth \& Tormen (1999) form for the halo mass 
function. We also integrate over a Bullock et al. (2001) concentration distribution at each mass, but suppress that integral in these equations for simplicity.

Our implementation of the galaxy trispectrum uses the subhalo HOD of K04. There are other parameterizations of the HOD, for example a binomial model by SSHJ, but the K04 model gives a simple form for moments of the HOD. The parameters of the K04 model are explained in Eq. (5), and the text above it.

The number of satellites is assumed to obey a Poisson distribution. This gives a simple formula for its factorial moments, which Kravtsov et al. (2004) found to hold through the third moment. Where $N_{s}^{(\alpha)}$ is the $\alpha$ th factorial moment of the satellite HOD,

$N_{s}^{(\alpha)}(m) \equiv\left\langle N_{s}\left(N_{s}-1\right) \ldots\left(N_{s}-(\alpha-1)\right) \mid m\right\rangle=\left\langle N_{s} \mid m\right\rangle^{\alpha}=\left(m / M_{1}\right)^{\alpha \gamma}$

The dark matter trispectrum has $1 \mathrm{~h}, 2 \mathrm{~h}, 3 \mathrm{~h}$, and $4 \mathrm{~h}$ terms, for which we refer the reader to $\mathrm{CH}$. When converting these to galaxy trispectrum terms, the $M_{\alpha}^{\beta}$ factors are replaced with $G_{\alpha}^{\beta}$, defined as

$G_{\alpha}^{\beta}\left(k_{1}, \ldots, k_{\alpha}\right) \equiv \int_{M_{\min }}^{\infty} u_{\mathrm{g}}\left(k_{1}, m\right) \cdots u_{\mathrm{g}}\left(k_{\alpha}, m\right) \frac{1}{\bar{n}_{\mathrm{g}}^{\alpha}}\left[N_{s}^{(\alpha)}(m)+N_{s}^{(\alpha-1)}(m) \sum_{i=1}^{\alpha} \frac{1}{u_{\mathrm{g}}\left(k_{i}, m\right)}\right] b_{\beta}(m) n(m) d m$.

This is a simplification of expressions by Smith. Watts \& Sheth (2007, e.g. their Eq. 76) for the case of the satellite HOD. Here, $\bar{n}_{\mathrm{g}}$ is the mean galaxy number density. For simplicity, we assume that the halo galaxy-number-density profile $u_{\mathrm{g}}$ is real, since we deal exclusively with spherically symmetric haloes. Ignoring the second term in brackets and the lower limit of integration, this expression has a nearly identical form to $M_{\alpha}^{\beta}$ in Eq. (B2). The second term in brackets shows the contribution by multiplets of galaxies with the central galaxy. The central galaxy is assumed to lie in the centre of its halo, so in real space, the term including it gets one fewer convolution over the galaxy-density profile.

For example, the galaxy power spectrum is

$$
\begin{aligned}
P_{\mathrm{g}}(k) & =P_{\mathrm{g}}^{1 \mathrm{~h}}(k)+P_{\mathrm{g}}^{2 \mathrm{~h}}(k)=G_{2}^{0}(k, k)+P^{\operatorname{lin}}(k)\left[G_{1}^{1}(k)\right]^{2} \\
& =\int_{M_{\min }}^{\infty} \frac{N_{s}^{(2)}(m) u_{\mathrm{g}}(k, m)^{2}+2 N_{s}^{(1)}(m) u_{\mathrm{g}}(k, m)}{\bar{n}_{\text {gal }}^{2}} n(m) d m+P^{\operatorname{lin}}(k)\left[\int_{\text {min }}^{\infty} \frac{N_{s}^{(1)}(m) u_{\mathrm{g}}(k, m)+1}{\bar{n}_{\text {gal }}} b_{1}(m) n(m) d m\right]^{2} .
\end{aligned}
$$

We note that expressions for the $2 \mathrm{~h}$ part of the halo-model galaxy power spectrum in the literature sometimes omit the second term in the $2 \mathrm{~h}$ numerator (the number 1 , which comes from pairs of central galaxies with each other), even though it dominates the first term in the $2 \mathrm{~h}$ numerator on small scales. However, the $1 \mathrm{~h}$ term is dominant over the $2 \mathrm{~h}$ term on small scales, so the central-galaxy contribution to the $2 \mathrm{~h}$ term usually contributes negligibly to the overall $P_{\mathrm{g}}$.

For the galaxy power spectrum and trispectrum, we do not change the galaxy-density halo profiles or concentrations from what we used for the dark matter. As with the matter covariance matrix, we integrate over concentration parameter at each mass. Galaxy-density profiles are almost certainly different from matter-density profiles in reality (e.g. Nagai \& Kravtsov 2005), but we are unaware of a simple analytic alternative to what we used for the dark matter. In any case, these approximations are adequate for our present purposes. 